\title{
Epidemiology: Gray Immunity Model Gives Qualitatively Different Predictions
}

\author{
Milind Watve ${ }^{1 *}$, Himanshu Bhisikar ${ }^{2}$, Rohoni Kharate ${ }^{3}$ \\ ${ }^{1}$ Independent researcher, E-1-8, Girija Shankar Vihar, Karve Nagar Pune 411052, India. \\ milind.watve@gmail.com \\ ${ }^{2}$ Indian Institute of Science Education and Research, Homi Bhabha Road,Pune 411008, India. \\ himanshu.bhisikar@students.iiserpune.ac.in \\ ${ }^{3}$ Independent researcher, 4, Shivratri Soc., Model colony, Shivaji nagar, Jail road, Nasik, \\ 422101. rohinikharate1712@gmail.com
}

*For correspondence: milind.watve@gmail.com

E-1-8, Girija Shankar Vihar, Karve Nagar Pune 411052, India 
Abstract:

Background: Compartmental models that dynamically divide the host population in categories such as susceptible, infected and immune form the mainstream of epidemiological modelling. Effectively such models treat infection and immunity as binary variables.

Method: We constructed an individual based stochastic model that considers immunity as a continuous variable and incorporates factors that bring about small changes in immunity. The small immunity effects (SIE) comprise cross immunity by other infections, small increments in immunity by sub clinical exposures and slow decay in the absence of repeated exposure.

Results: A model incorporating SIEs leads to qualitatively different epidemiological predictions including repeated waves without the need for new variants. Dwarf peaks, i.e. peak and decline of a wave much before reaching herd immunity threshold, low level apparently stable existence of the pathogen, new surges after variable and unpredictable gaps, new surge after vaccinating majority of population are the common features of the pandemic mimicked by simulations using the SIE model.

Discussion: The SIE model raises alternative possible causes of the universally observed dwarf peaks and repeated surges. We also suggest testable predictions to differentiate between the alternative causes. The model further shows complex interactions of different interventions that can be contextually synergistic as well as antagonistic. As a result, interventions intended to arrest the transmission can be ineffective or counterproductive. Interventions that are beneficial in the short run can be hazardous in the long run.

Conclusions: In the absence of empirical estimates of many parameters, the model may not be useful to make quantitative predictions at this stage but it certainly challenges traditional wisdom and currently held beliefs behind non-pharmaceutical interventions recommended to control the epidemic.

Keywords: Epidemiological model, dwarf peak phenomenon, herd immunity, Covid-19. 


\section{Introduction:}

Mainstream epidemiological models use a compartmental approach in which the population is dynamically distributed into compartments, typically three compartments namely susceptible, infected and removed (Tang et al 2020, Tolles and Luong 2020, Fudolig and Howard 2020) or at times more depending upon the question being addressed (Leung et al 2020, Giordano et al 2020, Carcione et al 2020). While this family of models is simple and useful towards some objectives, they are inadequate to capture certain features of real life epidemiological patterns as revealed by the Covid-19 pandemic (Moein 2021). Often the predictions of the model are empirically testable (Agarwal et al 2021). In the current pandemic some of the commonly observed features were not adequately explained by the mainstream models. During the Covid-19 pandemic in any of the surge, the peak appears to be achieved followed by decline substantially before reaching the herd immunity threshold expected at the estimated parameters. If anon pharmaceutical interventions (NPIs) or preventive restrictions (PRs) restricted the peaks, we should see significant and consistent decline in transmission rates after imposition of the measures. However, in data across countries the change in the slope is not correlated with standing stringency level and poorly correlated with change in stringency (Kharate and Watve 2021). This pattern is not adequately explained by the mainstream models so far. By classical model, the down-slope ends up approaching zero incidences, whereas during the pandemic it has commonly ended up in low and apparently steady level for quite some time before beginning of a consequent surge. The repeated wave pattern needs explanations outside the model such as change in behaviour of people as the fear reduces (Epstein et al 2021) or appearance of new variants (Fudolig and Howard 2020).

The compartmental models consider immunity as a binary variable. Any individual or a fraction of the population, when not suffering from active infection is either susceptible or immune in the model. We show here that considering immunity as a continuous variable rather than as a binary variable and incorporating subtle known factors affecting immunity explains many of the previously unexplained features of the epidemic. Allowing gray shades of immunity one can get repeated waves without involving new variants, increased rates of transmission without increased infectivity of the virus, a long term endemic steady state, a peak and decline much before the expected herd immunity threshold, breakthrough infections after vaccination and new surges after vaccinating majority of the population. The model that can explain these phenomena also 
gives certain non-conventional predictions that can be potentially important for designing control measures for future epidemics.

Incorporation of shades of immunity and dynamic population distribution of it necessitates an individual based model where the immunity level of each individual is affected by a number of factors. We only incorporate factors for which there is empirical evidence, at least qualitatively. Infection of an individual is a probabilistic phenomenon. In our approach immunity is measured in terms of the dose of infectious agent required to infect an individual with a probability of 0.5. The concept of ID50 is classical and well known (Antilla et al 2013, Regoes et al 2002, McLean and Bostoc 2000, Gadagkar and Call 2015) and the relationship between dosage of pathogen and probability of infection is of sigmoid nature with ID50 lying at the point of inflection. In our model we treat ID50 not as a population parameter but as a property of an individual depicting his probability of getting infected at a given level of exposure to the pathogen at a given time. Higher the level of immunity, higher is the ID50, i.e. higher the dose of pathogen required to cause infection. An individual's ID50 is a dynamic variable of the model that can vary between zero to infinity and that is under continued flux being affected by a natural infection or vaccination, which leads to a large quantum jump in ID50. This contrasts with many small immunity modulators. Some immunity is contributed as cross immunity from infection by other viruses in the form of antibodies to conserved epitopes (Mallajosyula 2021),interferons and other non-specific mechanisms (Dee et al 2021) and as a result some level of pre-immunity exists in a population before facing an epidemic (Sette and Crotty 2020, Doshi 2020, Ng et al 2020). A negative correlation between background level viral infection and the severity of Covid-19 pandemic is suggested by some studies (Haridas and Prathap 2020). Resistance to infection also involves subtle factors such as physical integrity of mucous membranes or other concerned tissues, the proportion of senescent cells in the target tissue (Humphreys et al 2020) and so on. After recovering from a respiratory infection, for example, much of the mucous membrane is composed of young cells that have replaced the infected cells. Such a young cell lining may be more resistant to a new virus, but this effect is expected to be short lived and wane fast as some older and effete cells accumulate. It is also possible that exposure to a pathogen at sub-infectious level contributes to some immunity (Gold et al 2021, Santos Rocha 2018), not comparable in magnitude to that achieved by active infection or by vaccines (Krammer 2021). Since immune response is costly, the body has evolved not to give a full strength immune response to every 
attack. Immune response is proportional to the intensity of invasion and virulence of the pathogen (Read 1994, Long et al 2020, Shinde et al 2021). Therefore the response to a mild exposure without detectable infection is likely to be proportionately small, but repeated exposures may raise the immunity level sufficient to increase the probability of escaping a given attack. In the absence of repeated exposure, immunity is known to decline gradually (Leino 2000, Sanderson 2021). This phenomenon was well recognized by previous models, but they still treated it with the binary immunity state assumption (Leung et al 2018). Different components of immunity are known to decline at different rates. For example, memory cells have a long life (Turner et al 2021) whereas antibody titres decline relatively fast. Some of the other non-specific small contributors mentioned above may also decline rapidly. At present there is little empirical work to parameterize these subtle factors. Therefore we do not incorporate the differential decline, but assume a small constant decline in ID50 with time in the absence of repeated exposure. The results of accommodating the small immunity effects (SIEs) are qualitatively different from the binary immunity models.

Owing to lack of empirical data on all the immunity related parameters, it is not possible to make a quantitatively predictive model for the Covid-19 pandemic at this stage. But our objective is to demonstrate that the SIEs can substantially affect the shape of an epidemic curve and therefore they demand more empirical inputs as well as a different class of futuristic models that will have a greater predictive value.

\section{The Model:}

We conceive an individual based model in which every $i^{\text {th }}$ individual in a population of $N$ has a dynamic immunity level ID5O $(i, t)$ which can change with time. There is a background population distribution of immunity levels before the epidemic begins which is assumed to be normally distributed initially with a mean ID50 (0) and s.d.ID50 (0). The nature of the distribution is allowed to change in time as individuals change their immunity levels. The probability of infection at a given level of exposure to the pathogen is assumed to be a sigmoid function by the classical principle of ID50 such that

$$
P_{\text {inf }}=\frac{E(i)^{a}}{\left(I D 50^{(i, t)^{a}}+E(i)^{a}\right)}
$$


Where $E(i)$ is the exposure bias (in arbitrary units) of $i^{\text {th }}$ individual to the virus. Individuals differ in their exposure to a pathogen by their profession, locality and behaviour and accordingly $E(i)$ also has a population distribution which is assumed to be constant in time. The parameter ' $a$ ' is the power that decides the sharpness of the sigmoid curve.

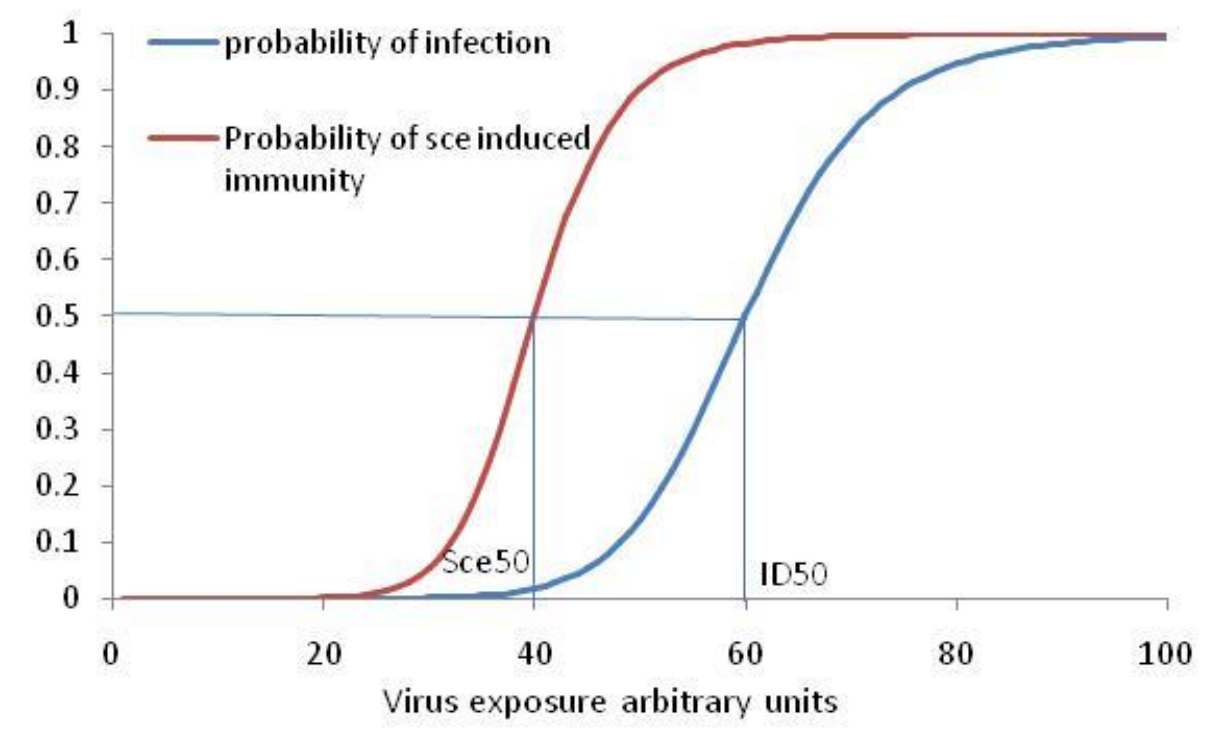

Figure 1: The sigmoid curve relating probability of infection as a function of exposure to infectious agent. ID50 or the point of inflection where the probability of infection $=0.5$ is a measure of standing immune level of an individual. We assume that exposure without causing infection contributes a small increment in immunity and this function is also assumed to be sigmoid.

$S(i, t)$ is a binary state of $i^{\text {th }}$ individual at time $t$ that can vary between $0=$ uninfected and $1=$ infected. With the probability $P_{i n f}$ the state changes from 0 to 1 . The infection is spontaneously cured with a constant probability $P_{c}$ by which a state 1 is transformed to state 0 and simultaneously $I D 50(i, t+1)$ is shot up by a factor $I_{\text {inf }}$.

We assume a small chance $P_{\text {cross }}$ with which an individual is infected by other viruses contributing cross-immunity. The level of cross immunity offered by such an infection is $I_{\text {cross }}$ assumed to be one to two orders of magnitude smaller than $I_{i n f}$. Similarly we assume that an exposure to the infectious agent without causing infection also confers a small immunity 
increment $I_{s c e}$ and this also works in a similar sigmoid function with $S c e 50(i)$ corresponding to probability of 0.5. Sce50(i) also has a population distribution which has a constant mean and s.d.

Whenever an individual's status is 0 and remains so, with neither $I_{\text {cross }}$ nor $I_{\text {sce }}$ increments, the immunity is assumed to decline by a small decrement $I_{d}$ which is much smaller than $I_{s c e}$ and $I_{c r o s s}$.

The exposure of individuals to the infectious agent also has multiple components and we include three important parameters representing them. One is the exposure bias of an individual. Some individuals by their occupation, type of housing, mobility and behaviour are more exposed and others relatively isolated. Therefore $E(i)$ is assumed to be the distribution of the exposure bias which remains time invariant. Exposure is also a function of the prevalent number of infectious individuals $I(t)$, but this need not grow linearly with $I(t)$. A society has some degree of viscosity and there is an upper limit $K_{c \max }$ to how many infectious persons one individual can come in contact. Therefore we consider the number of individuals from which a person can get infection as a saturating function of $I(t)$ such that,

$$
I_{c}(t)=\frac{K_{\text {cmax }} \cdot I(t)}{K+I(t)}
$$

The parameter $K$, the half saturation constant of the equation will be decided by population specific factors such as population density, mobility and behaviour of people.

The probability of an individual getting infected from at least one of the infectious contacts is

$$
\left.P_{\text {inf }}=1-\left[1-\frac{E(i)^{a}}{\left(I D 50(i, t)^{a}+E(i)^{a}\right)}\right)\right]^{I_{c}(t)}
$$

With this probability, the status of the individual at a given time $S(i, t+1)$ changes from 0 to 1 .

Similarly the probability that an individual gets a subclinical exposure that gives a small immunity increment $I_{\text {sce }}$ at time $t+1$ is

$$
P_{s c e}=1-\left[\left(1-\frac{E(i)^{a}}{\left(\operatorname{Sce} 50(i)^{a}+E(i)^{a}\right)}\right)\right]^{I_{c}(t)}
$$


We used stochastic simulations using these probabilities. The range of parameters used for these simulations is tabulated in table 1 . Note that the $I_{s c e}, I_{c r o s s}$ and $I_{d}$ ranges used for the simulation are one to three orders of magnitude smaller than $I_{i n f}$. Whenever we address vaccination related questions, the effect of vaccination is assumed to be equivalent to the effect of prior infection following Krammer (2021). Since distributions are being generated, we avoid zero and negative values by setting up an arbitrary small value to $E(i), I D 50(i, t)$ and $I(t)$. Any values going below this are considered equivalent to the minimum.

We used the model for running simulations (see supplementary information) with a range of parameters used as in table 1. Within the range one or more of the parameters were randomized. Since empirical estimates for many of the parameters are not available, we restrict ourselves to drawing qualitative conclusions that show alternative possible outcomes of any policy or measures implemented and do not claim any quantitative predictions.

Table 1:

\begin{tabular}{|c|c|c|}
\hline Parameter & Lower limit & Upper limit \\
\hline Mean $E(i)^{l}$ & 0 & 50 \\
\hline s.d. $E(i)$ & 1 & 30 \\
\hline mean ID50 $(0)^{2}$ & 0 & 200 \\
\hline s.d.ID50 (0) & 1 & 100 \\
\hline mean Sce50(i) ${ }^{2}$ & 10 & 50 \\
\hline s.d.Sce50(i) & 1 & 30 \\
\hline$a$ & 2 & 5 \\
\hline$P_{c}$ & 0.1 & 0.4 \\
\hline$I_{i n f}^{2}$ & 200 & 300 \\
\hline$I_{\text {cross }}^{2}$ & 0 & 40 \\
\hline$I_{s c e}{ }^{2}$ & 0 & 40 \\
\hline$I_{d}^{2}$ & 0 & 4 \\
\hline$P_{\text {cross }}$ & 0 & 0.1 \\
\hline$I_{\text {cmax }}$ & 5 & 40 \\
\hline$K$ & 50 & 2500 \\
\hline
\end{tabular}

1: Arbitrary units. 2. Immunity related parameters are expressed in the same units as 1. 
Simulating Non-pharmaceutical interventions (NPIs): During the Covid-19 pandemic, for the first time a set of NPIs were used on a global scale, consisting of certain preventive restrictions (PRs) imposed by government and personal protection measures (PPMs) advised to people. We addressed the effects of these on the epidemiological patterns to study their possible effects under different conditions. While the classical model only assumes that NPIs will reduce the parameter $\mathrm{R}$, our model allows exploring differential effects and interactions of different measures with each other and with the other parameters.

We expect NPIs to affect mean $E(i), P_{\text {cross, }} K_{c m a x}$ and $K$ to be affected by NPIs but in a differential way. Personal protection measures such as masks, PPEs, hand washing, surface sanitization and social distancing is expected to reduce meanE $(i)$ and $P_{\text {cross. }}$ Travel restrictions and work from home policy are expected to decrease $K_{c m a x}$ and/or increase $K$. We also consider a general lockdown parameter $L$ that goes from 0 to 1 and which multiplicatively alters $E(i), P_{\text {cross }}$ and $K_{c m a x}$ simultaneously and proportionately. These changes were applied either from the beginning of the simulations or at different $t$ representing imposition or relaxation of NPIs at different stages of the epidemic.

\section{Results:}

Since the model involves a large number of parameters we started with an exploratory approach to randomize all parameters within the given range (table 1) and record the qualitative outcome. Over a thousand simulations gave only four qualitative types of outcomes (figure 2),(i) A single peak followed by extinction or near extinction of the pathogen, similar to a typical compartment model (ii) a peak followed by a low level stable or mildly fluctuating endemic like state (ii) a pattern of multiple surges or waves, the surges often being separated by apparently stable or fluctuating incidence for variable time duration (also see figures 7,9 and 10) for variable spacing between waves). Since the number of parameters is large and we have no empirical estimates for many of them, we do not systematically explore the entire parameter space. Instead we focus here on demonstrating the complex interplay of parameters, the overall complexity of the outcomes and the inherent unpredictability of the system although certain qualitative pattern predictions can be made. This contrasts the classical deterministic predictive models and the change is brought about by making just one binary variable continuous. 


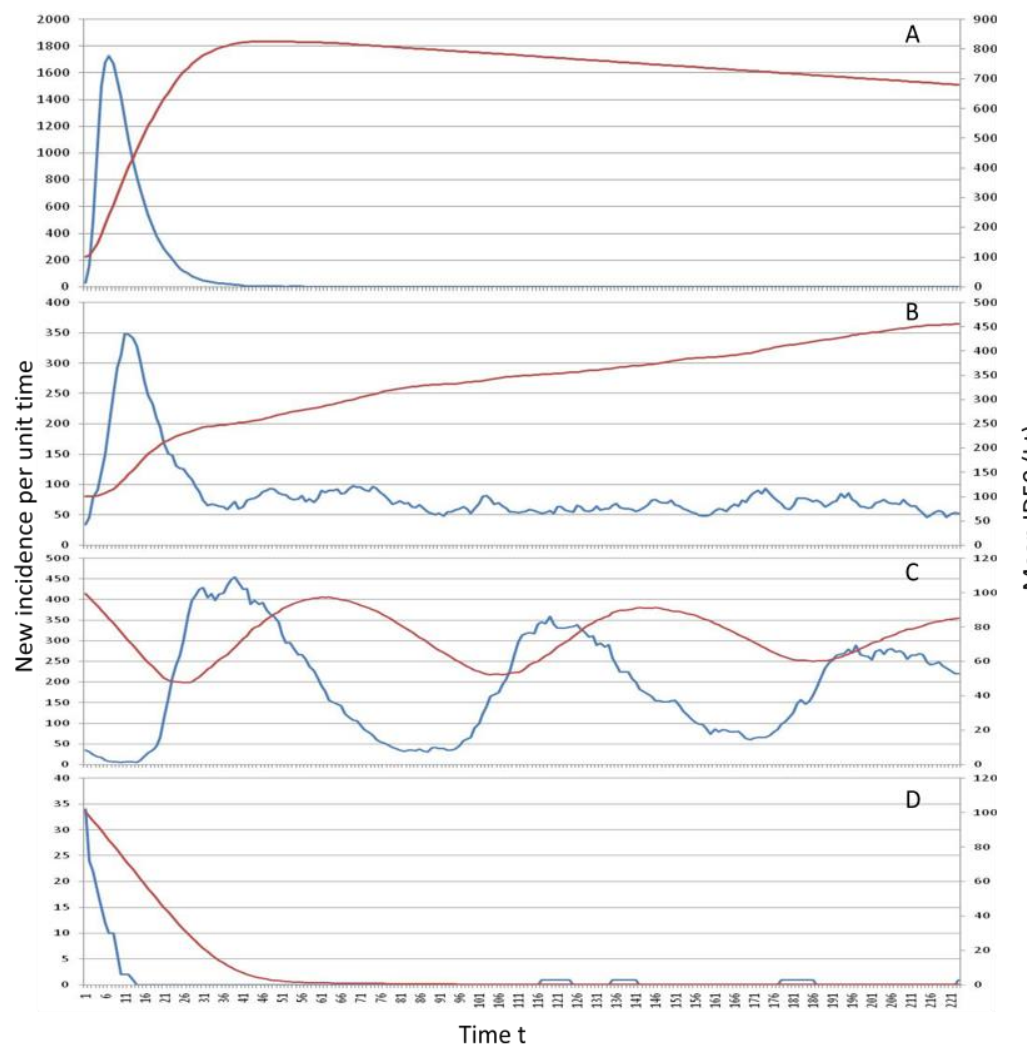

(Other parameters used in this simulation $N=5000$, mean $E(i)=$ $20 x L$, s. d. $E(i)=5$, mean $I D 50(I, 0)=100$, s.d. $I D(50)=30$, mean Sce50(i) $=20$, s.d. Sce50(i)=5, $P_{c}$ $=0.2, I_{i n f}=200, I_{s c e}=20, I_{d}=3$, $P_{\text {cross }}=0.1 x L, K_{\text {cmax }}=30 x L, K=2500$. In A. $L=1, B . L=.5, C . L=0.1, D . L=0$ and s. $d . E(i)=0.01$

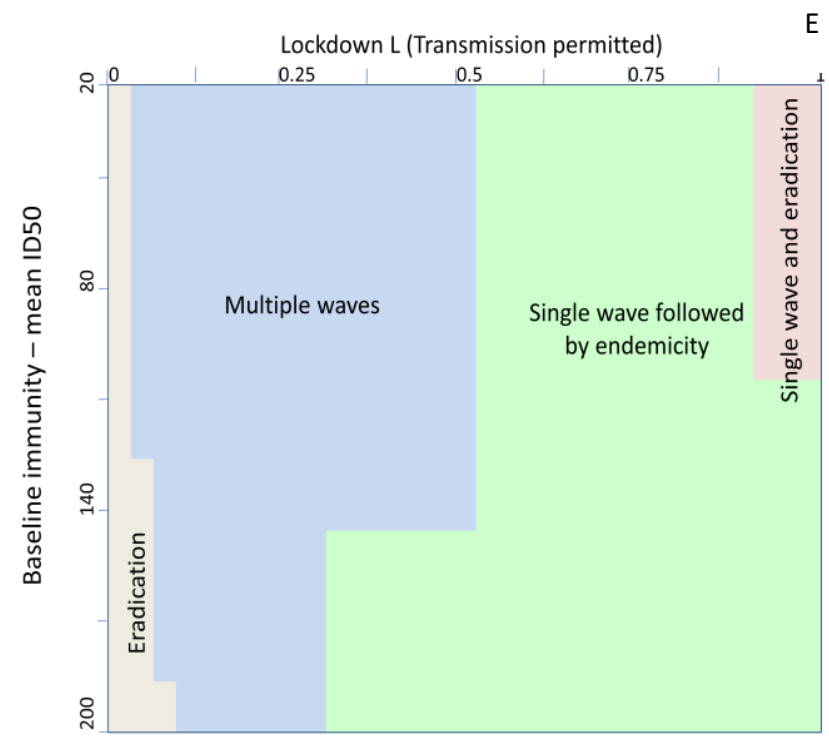

Figure 2: The four types of outcomes from the model. Blue lines = new cases per unit time, red lines $=$ mean population immunity level. A. Single wave followed by near eradication of the virus, B. Single wave followed by prolonged low level endemic coexistence C. Multiple surges with varying space between waves and heights of waves D. Effective control of transmission directly leading to eradication. E. An example slice of the parameter space across ID50(0) and L 
showing how the types of outcomes can be distributed. With high rate of transmission and low baseline immunity pattern A is observed. With greater restrictions on transmission endemic state ensues, further restrictions invite multiple waves but beyond a threshold restrictions, i.e. near zero transmission the disease may be quickly eradicated. The parameter areas vary widely as other parameters change but the four basic types are preserved.

When the small immunity effects are zero or close to zero, the outcome is similar to classical compartment models implying that the apparently stable endemic or multiple well spaced wave patterns are a result of the SIE. It is notable that in this model when SIE parameters $>0$, the height of a peak and subsequent decline begins when only a small fraction of the population is infected, in contrast to the herd immunity threshold of classical SIR model. An important outcome of the model is that a wave pattern with variable spacing, slopes and heights of the waves is possible even without the need for novel variants defying immunity.

Although the system assumes a state of complexity with limited quantitative predictability, the processes leading to the four possible outcomes can be explained in principle. Owing to the sigmoid relationship of infection probability with the dynamic individual ID50 values, at times a small increase in immunity can be sufficient to evade infection with a high probability. The escape is likely to be accompanied by further small increase in ID50 reducing the probability of infection further. By this mechanism, many individuals can escape infection without being "fully" immune. This results into an arrested peak followed by decline in incidence much before the classical herd immunity threshold could be reached. One of the patterns predominantly observed during the first phase of the pandemic was that most members of the family of infected individuals appear to have escaped infection in spite of exposure (Shah et al 2020). This can also be explained by the SIE effect. However, as the incidence declines, the immunity levels achieved by SIEs also start declining. The rate of decline is enhanced by NPIs. This makes some of the individuals susceptible again. This leads to a complex dynamics resulting into a fluctuating incidence that may remain apparently stable for some time or give rise to another surge when the immunity levels of a substantial part of the population decline below a threshold.

The immunity decline is more relevant to individuals with smaller $E(i)$ as they remain protected owing to lower exposure. However, as they remain protected, their immunity also declines slowly. With the decline, they become increasingly susceptible at a lower exposure. This is 
unlikely to happen to individuals who achieve immunity after an active infection or by vaccination. This is because the rise in immunity is of a much greater magnitude than the SIEs. Although the infection or vaccination acquired immunity is also subject to waning, during the long time required for waning they have a greater chance of repeat subclinical exposures boosting the immune levels again. Therefore the second wave is mostly due to decline in the SIE rather than decline in infection or vaccination driven immunity. This is also evident in the gap between two surges which can be often much smaller than $I_{\text {inf }} / I_{d}$.

When the rate of a process is determined by multiple factors, only some of them are rate limiting at a given set of conditions. Therefore only intervention in these factors can result into effective control of transmission. Intervention in factors that are not rate limiting is not likely to have any effect. The factors interact with each other in a complex way, sometimes being synergistic but at other times working to nullify each other's effects. Simulations using our model demonstrate this well known principle, some examples being depicted in figure 3 .
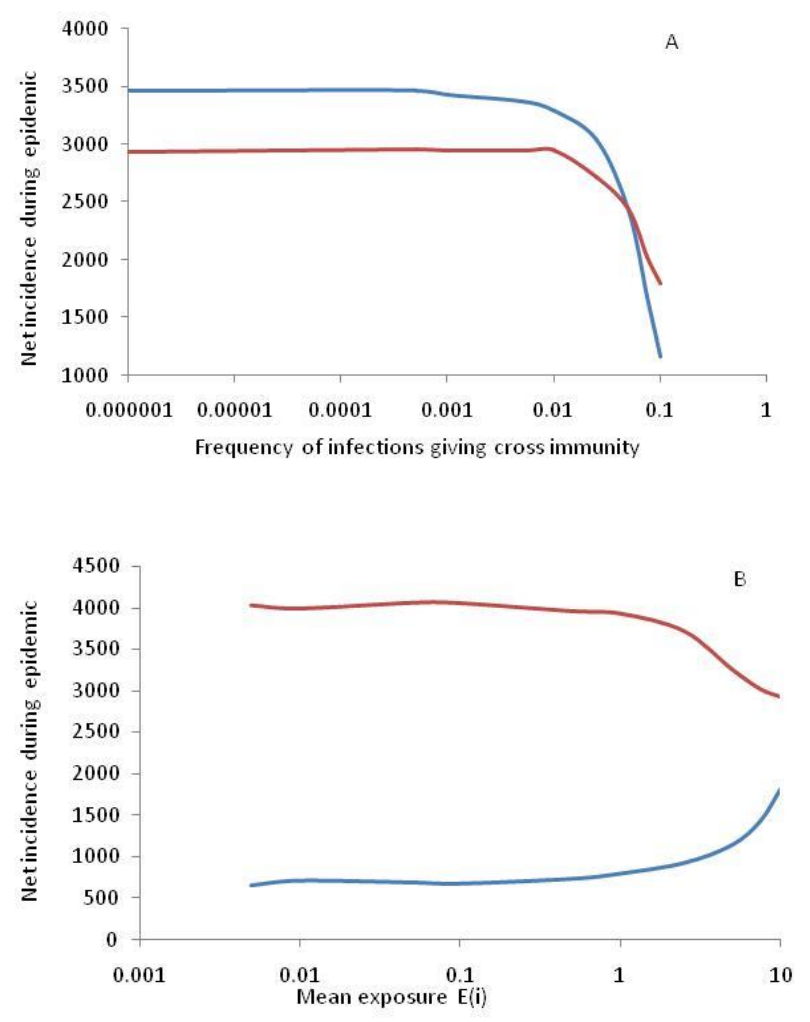

(Other parameters used in this simulation $N=5000$, mean $E(i)=10$, s. $d$. $E(i)=5$, mean ID50(I,0)=100, s.d. $I D(50)=60$, mean Sce50(i) $=20$, s.d. Sce50(i)=5, $P_{c}=0.2, I_{\text {inf }}=200, I_{s c e}=20$, $I_{d}=2, K_{\text {cmax }}=30, K=2500$. 


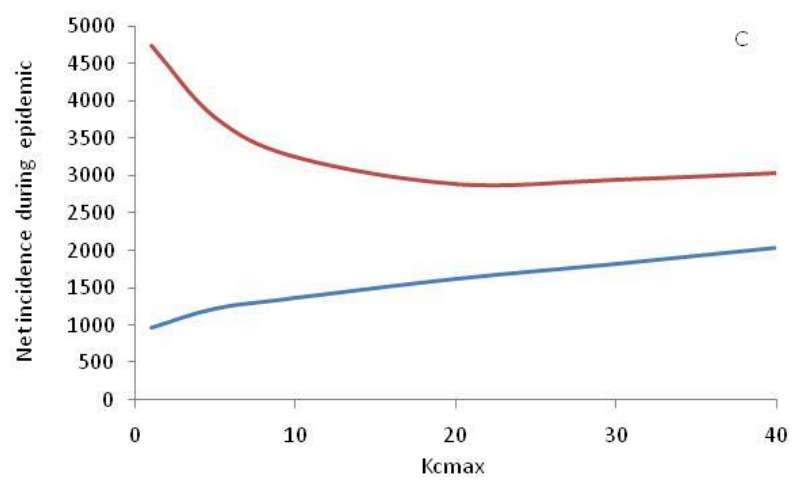

Figure 3: The complex nature of interaction between the parameters affecting net incidence during the simulated pandemic A. Effect of $P_{\text {cross }}$ at two different mean E(i), 5 (blue line) and 10

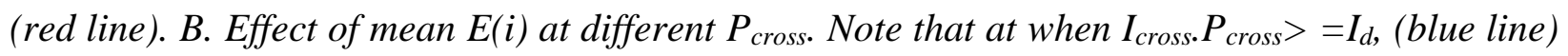
reducing mean $E(i)$ reduced the incidence whereas at $I_{c r o s s} P_{c r o s s}<I_{d}($ red line) reducing $E(i)$ increased the net incidence. $C$. Effect of $K_{\text {cmax }}$ at $I_{\text {cross. }} P_{\text {cross }}>=I_{d}$, (blue line) and $I_{\text {cross. }} P_{\text {cross }}<I_{d}$ (red line). Awork from home strategy that reduces $K_{\text {cmax }}$ may reduce the incidence in the former condition whereas work from home can actually increase disease incidence in the latter. The examples illustrate complexity of interactions between factors owing to which the measures intended to reduce transmission can be effective, ineffective or even counterproductive in different contexts.

As a result of the complex interactions, different factors become rate limiting in different phases of the epidemic and a measure that is effective in one phase may prove ineffective in another. For example in one phase of the epidemic the rate of transmission of the virus may be central in determining the slope of the incidence curve whereas in another phase the rate of immunity decline may be the limiting factor and a change in rate of transmission may bring in no detectable change in the dynamics (figure 4). It is important to realize this in planning public health policies. The complex interactions possibly explain the lack of consistent relationship between various PRs imposed and the change in slope of the incidence curve to follow. Imposing a restriction is expected to decrease the slope and lifting imposition is expected to increase it. But in data across countries we observe that contrary to expectation $31 \%$ of times slope actually increased after imposing PRs and $45.4 \%$ of times it decreased after relaxing them (Kharate and Watve 2021). A simple but highly enlightening possibility raised by the model is that the simple assumption that PRs decrease disease transmission is not always true. 
A.

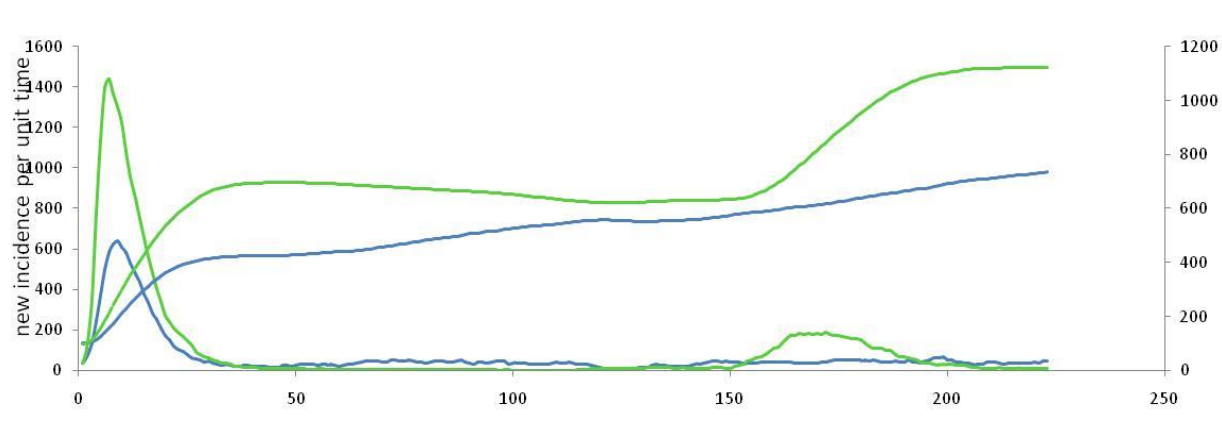

(Other parameters used in this simulation $N=5000$, mean $E(i)=10$, s. $d$. $E(i)=5$, mean $\operatorname{ID} 50(I, 0)=100$, s.d. $I D(50)=30$, mean Sce50(i) $=20$, s.d.Sce 50(i)=5, $P_{c}$ $=0.2, I_{i n f}=200, I_{s c e}=20, I_{d}$ $=2, P_{\text {cross }}=0.1, K_{\text {cmax }}=30$,

B. $K=2500$.

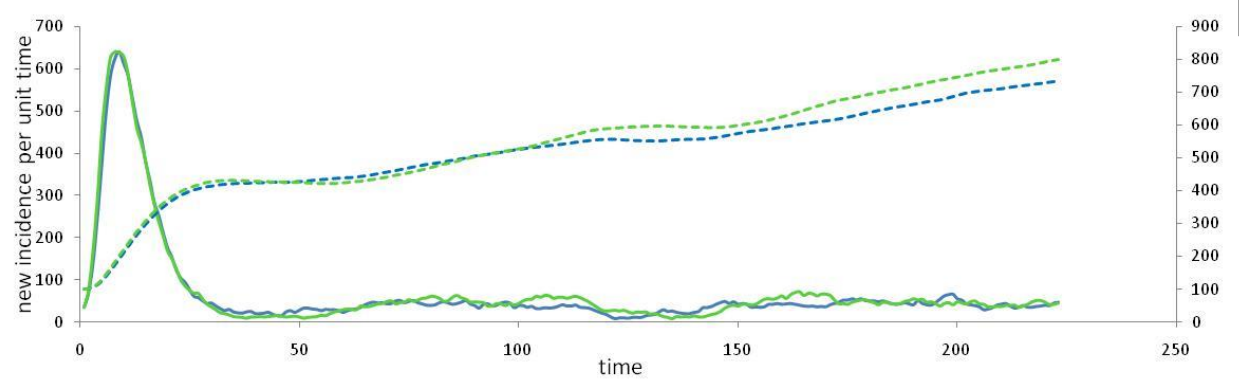

Figure 4: The effect of the same intervention at different phases of the epidemic can be very different. (A) Two exposure levels mean $E(i)=15$ (green lines) and 10 (blue line) from the beginning of the simulation $(B)$ Two simulations started with mean $E(i)=10$ but at $t=100$ one of them changed to 15. Note that controlling exposure from $t=0$ substantially lowers the incidence, but relaxing the control at a later phase, $t=100$, has no significant effect on the course of the epidemic. A possible implication of this is that if a new variant with greater infectivity arises during the endemic like state, the increased infectivity alone may not be sufficient to cause another peak. Also relaxation of PRs may increases $E(i)$ but that may not result in rise in incidence.

We see in the Covid-19 data across different populations that most of the peaks have been much smaller than the herd immunity thresholds that were predicted. This is compatible with our model. However there are two possible alternative explanations for having dwarf peaks. One is that the non-pharmaceutical interventions (NPIs) or preventive restrictions (PRs) effectively restricted the transmission and arrested the peaks. The other is that most individuals could escape infection owing to the SIE effects restricting the peak height. It is possible to make differential predictions from the two alternative hypotheses for the small peaks. If the preventive restrictions 
arrest the infection and turn the curve downwards by effectively making $\mathrm{R}<1$ in the classical model, the slope of the downward curve is expected to be independent of the upward curve (figure 5a). On the other hand, in the classical model, as well as in our model when the curve naturally starts a decline owing to altered level of population immunity, there remains an element of symmetry in the shape of the peak. If the upward slope is steeper, the downward slope is also proportionately steeper. This is because a rapid rise in incidence also causes a rapid rise in immunity by the classical model as well as by SIE effects and a greater level of population immunity causes a steeper decline. This leads to a good correlation between the upward and downward slopes as revealed by simulations (figure 5b and c). Applying PRs at some point in a rising wave, and assuming that the PRs are effective, a decline before the herd immunity levels can also be obtained. But in this case the slope of the decline is driven by the intensity of the PRs and therefore may not be related to the upward slope before the imposition of PR. High population density and other pro-transmission factors that are responsible for a steep upward slope, make a steep downward slope more difficult. This is most likely to deviate further from the symmetry of the wave. Therefore using a correlation between the upward and downward slope it is possible to test on a global scale whether PRs were commonly responsible for the shorter peaks or SIE effects. A careful analysis of symmetry of peaks (See supplementary material for details of symmetry analysis) reveals highly significant correlation between upward and downward slopes (figure $5 \mathrm{D}$ and $\mathrm{E}$ ) of all major peaks indicating substantial role of SIE and not NPIs alone.

Figure 5: Differentiating between two alternative causes for dwarf peaks: In the incidence curves of Covid-19 across countries, it is observed that almost all the peaks are achieved much before the predicted herd immunity level. This can either be because of the preventive restrictions imposed or because of the small immunity effects. A. In a classical SIR model, the $P R$ s are expected to make $R<1$ if a decline is caused by them. However, they may take any value between zero and one independent of the upward slope of the surge. Therefore no correlation is expected between the upward and downward slope. B. Simulations using our model in which the parameters are randomized to get variance in transmission rates, show that a steep upward slope is accompanied by a steep downward slope giving rise to a correlation between the two (C). In pandemic data across countries covering all 164 peaks of height over 1000 (new cases per day) there is good correlation between upward and downward slopes taken over linear scale 
( $r=-0.861$ including second peak of India which is an outlier and 0.599 excluding it) as well as log scale ( $r=-0.4304)$. For all the three correlations $p<10^{-6}$.

A.

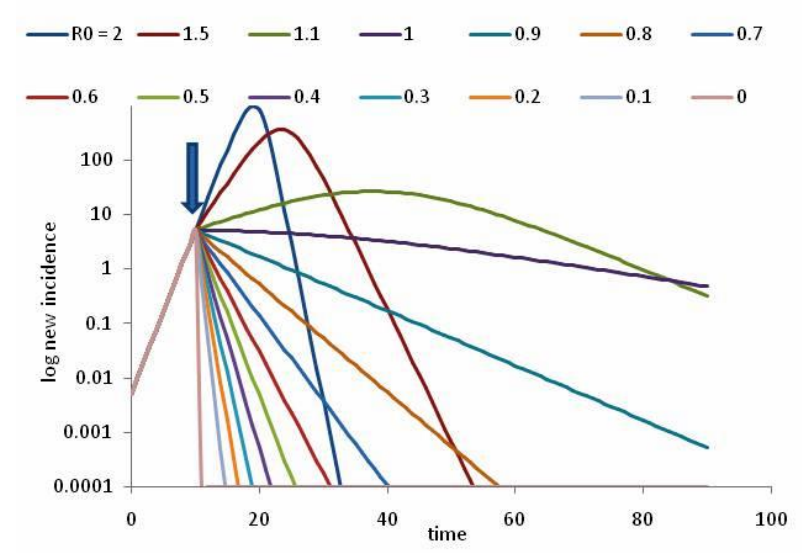

C.

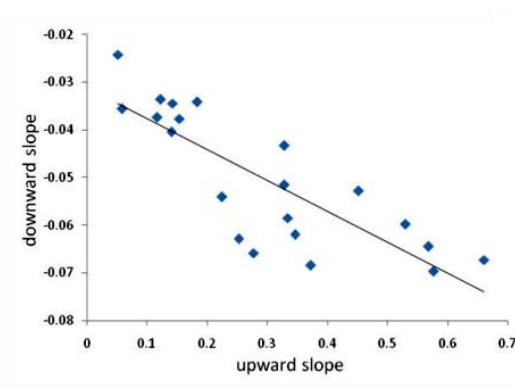

B.

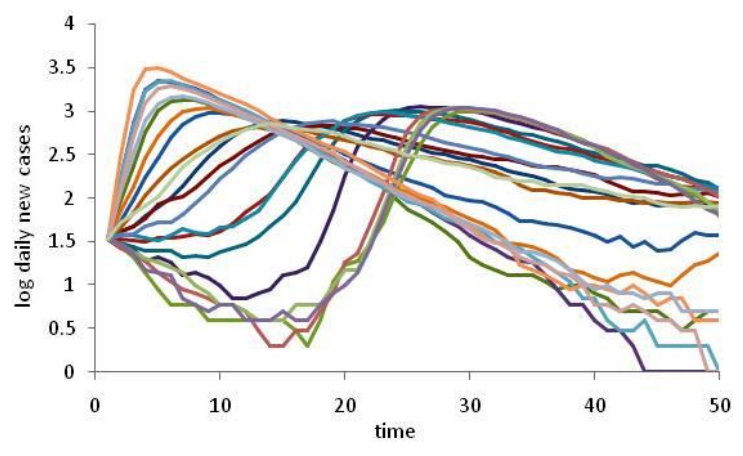

E.

The starting ID50 distribution in the population is assumed to be normal in the SIE model. However, the shape of the distribution will change as the epidemic progresses. While infected and recovered individuals will have experienced a quantum jump in their $\operatorname{ID50}(i, t)$ the protected part of the population may have lost some, particularly if the personal protection measures are strictly followed. Further as a wave recedes, exposure becomes rarer and the immunity is gradually lost for a larger sector of the population. The distribution of immunity in the population at this stage is highly dispersed and often bimodal (Figure 6). Although the individuals having acquired immunity after an active infection will experience little proportionate loss and will be least susceptible to reinfection for a longer time, the once having escaped because of SIE may have reduced immunity as compared to their baseline particularly if 
personal protective measures have been strictly followed. The resulting bimodal or over dispersed distribution of immunity has important consequences for the shape of the incidence curve. The pathogen may persist in the population without becoming extinct owing to newly created susceptibles that can get infected at very low exposures. If the immunity of a substantial sector of population drops below a threshold, a new wave may begin. Therefore depending upon the parameters and the standing context, the epidemic can take wave forms without the need for new variants or behavioural change in the population. The possibility of variably spaced repeated waves is a unique outcome of the SIE model not seen with the classical compartment models without involving novel variants or recruitment of new born susceptibles.

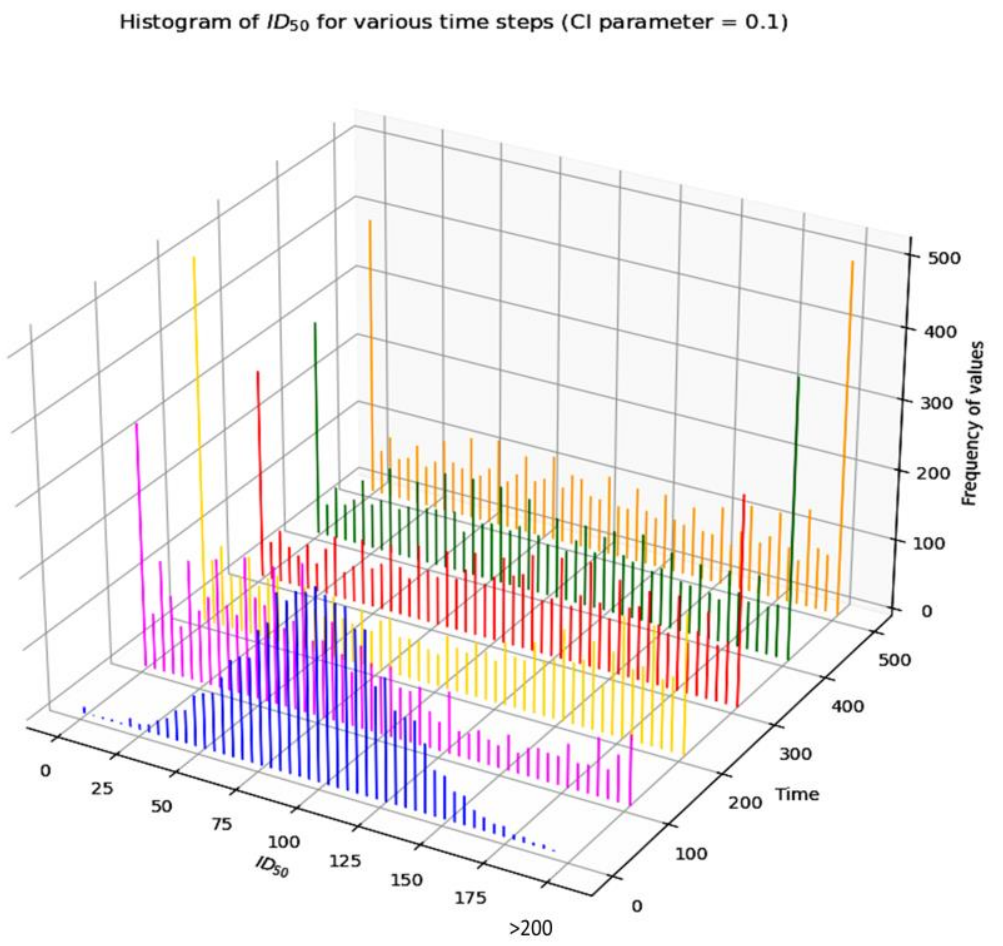

Figure 6: Change in the distribution of population immunity during the course of the epidemic when NPIs are applied but are unable to eradicate the pathogen in one go. The assumed normal distribution of immunity in the beginning of the epidemic becomes increasingly over-dispersed. The immunity levels decline starting with the protected sector of the population (low E(i)) and when near the lower end, some of them get infected even at very low exposures. These individuals are thrown to the right extreme because of infection induced immunity, but 
simultaneously more individuals lose immunity to take their place. This immunity dynamics appears to be mainly responsible for the stable or oscillating incidence.

Nevertheless the model is not incompatible for new variants which might escape the specific immunity to earlier variant or may be mutants with higher infectivity. It is possible that both SIE and new variant contribute to repeated waves. In the context of repeated waves, another interesting result of the SIE effect is that even in the absence of a new and more infectious variant, the second wave can be steeper and taller than the first one (figure 7). This happens when initial population immunity, mean $\operatorname{ID} 50(i, 0)$ is substantially greater than mean $E(i)$, both the distributions have sufficiently large standard deviations and personal protection or general lockdowns are sufficiently effective to allow loss of immunity in a sector of the population. Under such conditions the first wave is mainly limited by pre-existing cross-immunity of the population. However, due to personal protection measures exposure to other viruses giving cross-immunity is also reduced for the majority of the population. As a result the distribution of immunity becomes bimodal and for substantial population the immunity declines below a threshold to trigger a new wave. Since now the background immunity for a sector of the population is lower than the first wave, the second wave rises more sharply and achieves a greater height. Such a pattern is seen in many countries during the current pandemic. This creates an alternative possible explanation to the more devastating second wave faced by countries such as India. The first wave in the highly population dense India was said to be surprisingly small with low mortality which could be ascribed to a background immunity level contributed by other viral infections (Haridas and Prathap 2020). However owing to widely applied lockdown and personal protection measures, the immunity of a substantial sector of the population could have declined, making them highly susceptible. This susceptibility could be the cause of the steeper and more devastating second wave. Currently the devastating nature of the second wave in India is attributed to the variant Delta (Mallapaty 2021b) without considering the alternative hypothesis of SIE. The two possible causes of the second wave being more devastating are not mutually exclusive, but it is necessary to analyze and understand their relative contributions by a differential predictions approach. 


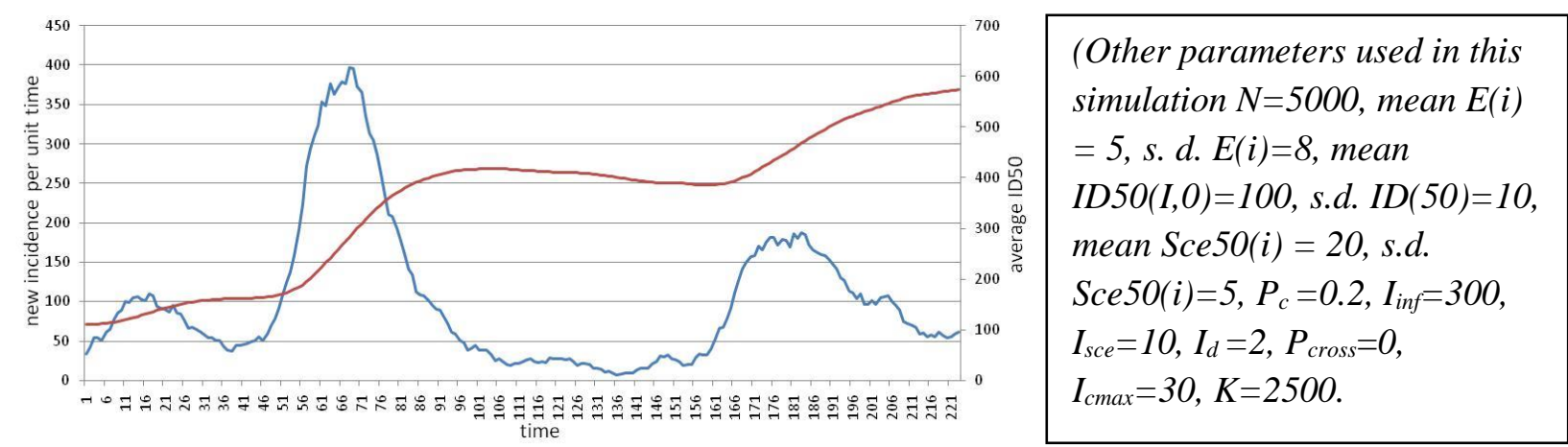

Figure 7: The phenomenon of second wave being steeper and taller than the first can be obtained in the SIE model without the need for a more infectious variant. The steepness of the second wave is caused by the subtle accumulating immunity loss intensified by NPIs. Increasing one or more of the exposure related variables from the start of the simulation increases the first peak and suppresses the second.

The alternative causes of the second and subsequent waves can be differentiated based on the epidemiological patterns they give rise to. The possible causes of second wave can be different if the first peak has achieved herd immunity or is a dwarf peak due to SIE effects or due to NPI. If a new variant evades immunity against the prior variant, a new wave may begin but the pattern of this wave with respect to individual exposure bias will remain similar to the first wave, i.e. individuals with greater risk of exposure will be affected first. If the first wave is dwarfed by NPIs, in the absence of SIEs, a novel antigenic variant will be unable to cause a second wave unless the NPIs are relaxed and/or the infectivity is increased. In simulations increasing infectivity or relaxing restrictions is treated similarly by increasing the exposure related parameters. In simulations if SIEs are disabled and the first peak is substantially dwarfed by NPIs, the exposure, infection relationship is positive as in the first wave. Even if immunity evading variant and increased infectivity act together, the positive relationship between exposure and infection is retained in the second wave. In contrast, if the first peak is dwarfed by SIEs a second peak can arise without a new variant and in that case, the low exposure individuals are more likely to be infected in the second wave (Figure 8). 


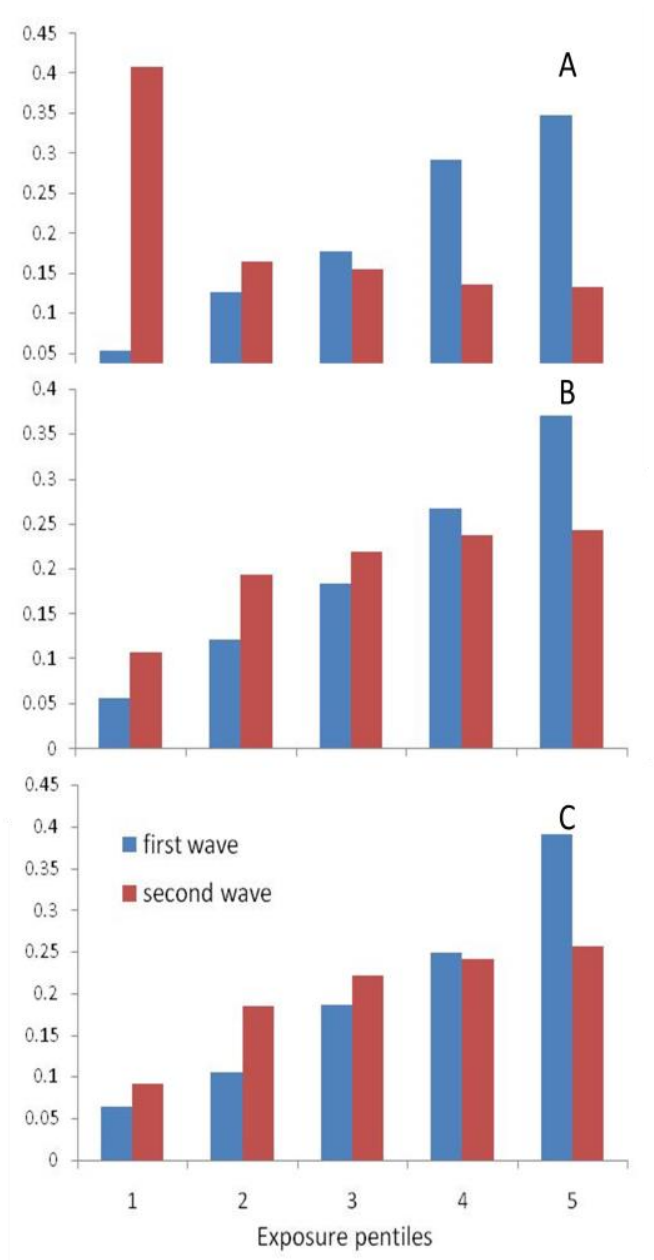

Figure 8: The exposure infection relationship during the first and second wave: Relative frequency of infected individuals belonging to the five pentiles of the exposure distribution when the second wave is caused by (A) SIE model, (B) variant with greater infectivity or restrictions relaxed and (c) variant with greater infectivity as well as partial immunity evasion. In the SIE model the relationship is reversed during the second wave, i.e. individuals less exposed to the first wave are more likely to get infected in the second. In contrast if the second wave is caused by lifting restrictions or a new variant with higher infectivity and/or evading immunity to earlier variant the exposure-infection relationship is not inverted.

This contrasting pattern is testable in real life. Although precise quantification of individual exposure bias may be difficult, it can be predicted that if SIEs cause a first dwarf peak followed by a second wave, individuals whose occupation exposes them more to infected individuals, who live in population dense locality and/or do not follow appropriate behaviour will be infected 
disproportionately more in the first wave whereas in the subsequent waves individuals from more isolated, remote and low density areas, with safer occupations and following Covid appropriate behaviours will show disproportionately increased relative incidence.

The bimodality of immunity in the SIE model also suggests a possible cause of the observed incidence surge in many countries after nearly half or more of the population getting vaccinated. If vaccination is accompanied by effective personal protection measures, while the vaccinated individuals have high levels of immunity, the unvaccinated section of the population might have reduced their immunity levels substantially triggering a new wave. This is seen very frequently in simulations if we introduce vaccination in a part of the population at some point after the first wave has declined (figure 9).

In the simulation we consider the vaccinated individuals to be completely immune. If vaccination does not completely prevent infection but arrests severity, vaccinating part of the population can create a much stronger surge because of a combination of infected but not seriously ill individuals among the vaccinated and increased susceptibility among the unvaccinated. The effect of vaccination also depends upon at what time mass vaccination begins. If it begins after prolonged lockdown it is likely to be less effective since the baseline immunity itself has declined. Also repeated exposure after vaccination is likely to boost immunity further and keep it high, as documented earlier for other viral infections (Whittle 1999). Lockdown is therefore likely to be partially antagonistic to vaccination.

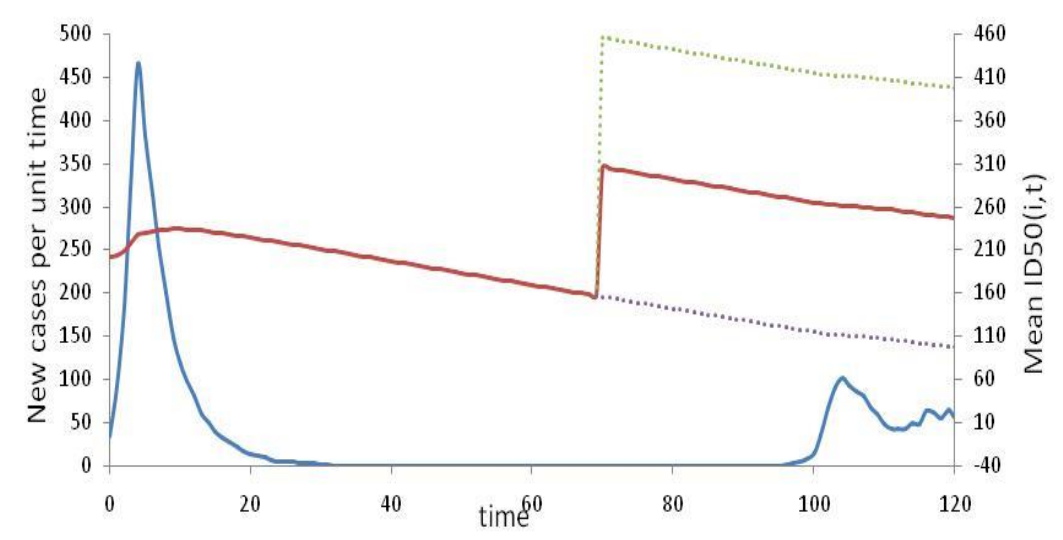

(Other parameters used in this simulation $N=5000$, mean $E(i)=20$, s. $d$. $E(i)=15$, mean $\operatorname{ID} 50(I, 0)=200$, s.d. $I D(50)=70$, mean Sce50(i) $=20$, s.d. Sce50(i)=5, $P_{c}$ $=0.2, I_{\text {inf }}=300, I_{s c e}=10, I_{d}$ $=1.5, P_{\text {cross }}=0.05, I_{\text {cmax }}=30$, $K=2500$.

Figure 9: A surge of variable intensity is possible after vaccination of a substantial proportion of the population. Here at $t=75,50 \%$ of the population is assumed to be vaccinated instantly. 
Although the mean immunity of the vaccinated sector goes up (dotted green line) pushing the average up (red line), the unvaccinated ones continue to lose immunity slowly (dotted violet line) and after a threshold loss a new surge may begin.

The model finds its most important use in predicting the qualitatively different possible outcomes of PRs. The generalized outcome is that the effects of PRs can be non-monotonic and depend substantially on the context. What can be good in the short run may turn counterproductive in the long run (figure 10a). Also the stringency of PRs may not be linearly or even monotonically related to the total incidence (figure 10b) as observed commonly in simulations over a large parameter space.
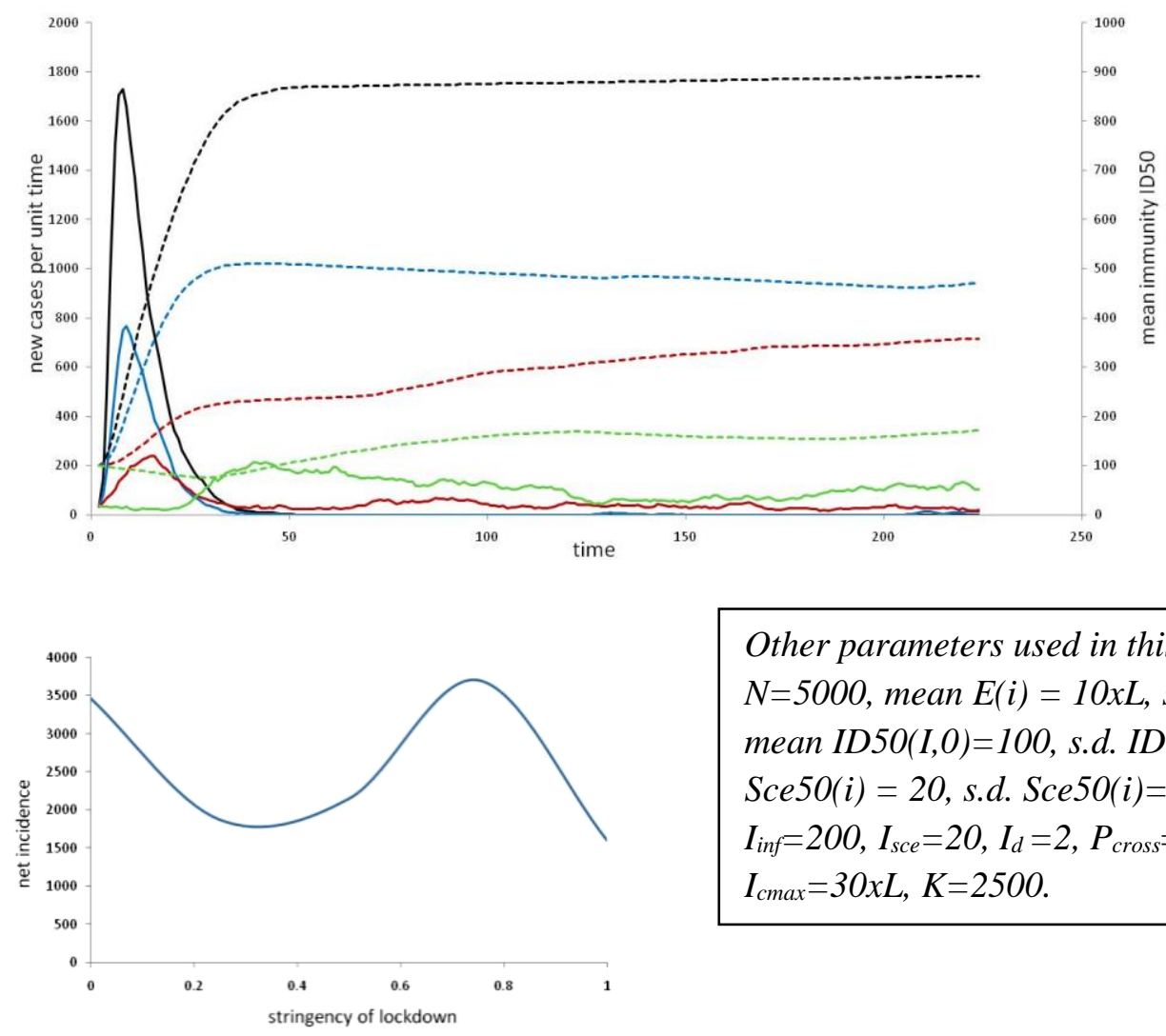

Other parameters used in this simulation $N=5000$, mean $E(i)=10 x L$, s. d. $E(i)=5$, mean ID50(I,0)=100, s.d. $I D(50)=30$, mean Sce50(i) = 20, s.d. Sce50(i)=5, $P_{c}=0.2$, $I_{\text {inf }}=200, I_{s c e}=20, I_{d}=2, P_{\text {cross }}=0.01$, $I_{\text {cmax }}=30 x L, K=2500$.

Figure 10: The non monotonic effects of PRs: Course of an epidemic at different values of $L$ between 0 and 1. As the lockdown stringency increases, the first peak is suppressed, but post peak incidence is maximum for intermediate values of $L$. As a result, the net incidence over the course of the epidemic is non-monotonic. Stringency of lockdowns may not be beneficial and may turn out to be counterproductive at certain levels. 
Since we have little information about the SIE related parameters, it is difficult to predict whether PRs will be beneficial or hazardous in different populations at different times.

\section{Discussion:}

An epidemic disease is a complex system which is difficult to predict quantitatively as witnessed during the ongoing Covid-19 pandemic. The predictions of the current mainstream modelling based on compartmental models largely failed in making qualitative as well as quantitative predictions. Not only the number of cases or number of deaths could not be predicted, but that there would be repeated waves, the causes, the timing and the severity of waves also could not be predicted at a level significantly above common sense. This is likely to be an effect of oversimplifying a complex system. Modelling is best done with an incremental approach. When a simple model fails to meet its objectives, it is necessary to incorporate crucial elements of the complexity into the model. What we show here is that treating immunity as a binary variable needs to be replaced by treating it as a continuous variable and incorporating small effects contributing positively and negatively to immunity. Allowing gray shades of immunity alone makes the model display characters of a complex system and brings in many qualitative changes in the model outcomes. Potentially even infection need not be treated as a binary variable since the intensity of infection matters. The immune response is proportionate to the intensity of infection (Long et al 2020, Shinde et al 2021, Read 1994) and the standing level of immunity is expected to affect the intensity of infection thereby the two continuous variables can have a complex interaction. Our model has also not incorporated severity of symptoms and mortality. In an epidemic the viral infectivity and virulence is also subject to natural selection and changes during the course of the epidemic are inevitable (Shinde et al 2021). The transmission dynamics depends upon season and local variables such as population density, type of housing and workplace, nature of transport, weather conditions that differ from population to population and time to time. This makes the entire system highly complex. Predicting a complex system by pretending it to be simple, not only makes the predictions dicey, it severely limits the thinking in the domain including the qualitative perception of non-modellers. The small immunity effects modelled here are not unknown. But the current epidemiological qualitative thinking also does not incorporate them possibly because starting the model with a simple set of assumption also makes the non-modellers forget that processes not currently incorporated in the model do exist. 
A gray immunity model is an important step in the incremental approach and our simulations show that it can potentially explain the short and self limiting peaks, repeated surges with increasing or decreasing amplitude and variable gaps between them, an apparently stable endemic like state, surges even after vaccinating majority of the population. All these phenomena are seen in simulations without necessarily involving new variants. Further we also make differential testable predictions to resolve between alternative causes of these phenomena. The most important outcome of the gray immunity model is the possibility that the long term effects of preventive restrictions can be non-linear, non-monotonic and the interventions that can be beneficial in the short run can be counterproductive in the long run, a warning bell already rung by Hollingsworth et al (2020) in the context of other infectious diseases. In this connection, it should be noted that a WHO (2019) report based on meta-analysis of several studies published just before the beginning of the pandemic showed that most of the NPIs for respiratory infections have little evidence of being effective. All the studies claiming success of NPIs in arresting infection (e. g. Alfano and Ercolano 2020, Kharroubi and Saleh 2020, Atalan 2020, Brauner 2020, Abaluck et al 2021) are short term studies and therefore whether the short term benefit has turned counterproductive during the epidemic has not been studied.

Our assumptions about small immunity effects are not without empirical support. The immunity boosting effect of repeated subclinical exposures is well demonstrated for other viral infections such as measles (Whittle 1999). In the Covid-19 pandemic our assumption that NPIs reduce other common respiratory infections is evident in data (Marriott et al 2021, Huang 2021). It is also argued that by inhibiting common respiratory viruses the general respiratory immunity in the population may have declined substantially (Cohen 2021).

Of particular interest is that the model gives a possible alternative causal hypothesis to the dwarf peaks and repeated waves which are currently believed to be caused by differential implementation of PRs and by new variants respectively. The three factors are not mutually exclusive and could be acting together or in complex interactions. In the light of the gray immunity model it is possible to take a perspective of an analytical as well as accommodative causal analysis. There are two possible reasons for the dwarf peaks. The currently believed one is that the PRs restricted the peaks and the alternative explanation is that most individuals escape infection owing to small immunity increments. We have already stated the differential 
predictions of the two. According to the PR hypothesis, there should be no correlation between the upward slope and downward slope of the peaks which is expected to be quite strong by the SIE hypothesis. In reality most peaks are highly symmetrical as shown by the highly significant correlation between upward and downward slopes. In addition, the analysis of change in slope of the incidence curve following imposition or relaxation of PRs has already shown that the effects of PRs are marginal (Kharate and Watve 2021). Differentiating between the causes of dwarf peaks is important because arguments such as the lockdowns saved so many deaths (Agarwal et al 2021) are based on the assumption that peak heights were restricted by PRs. If the dwarf peaks are a result of SIEs, then the estimates of the effects of lockdowns () are bound to be gross overestimates.

The appearance of repeated waves can have three possible causes. One is that of fluctuating stringency of PRs and changing behavior of people. When the incidence curve is upwards and mortality is high, PRs are imposed and people follow behavioral guidelines under the fear of the infection. When incidence is low, restrictions are relaxed and fear is lowered which together lead to increased transmission again. This hypothesis assumes that PRs were highly effective. But the small and inconsistent changes in slopes after imposing PRs and the symmetry of the peaks weakens the PR induced dwarf peak hypothesis. The second prevalent explanation is that each new wave is caused by a new variant that is either capable of escaping immunity and/or more infectious than the earlier variant(s). The SIE model has raised a third possibility that repeated waves are intrinsic to the small immunity effects over a wide parameter space. It is possible to make differential testable predictions of the three.

A new variant can cause a new wave if it can evade the immunity against the prior variant(s). If this is qualitatively true, immunity acquired by prior infection or vaccination becomes irrelevant individuals with a higher $E(i)$ would be more prone to infection in the first wave as well as second wave. On the contrary, by the immunity loss hypothesis, second wave is expected to affect more of the class that remained relatively protected in the first wave, i.e. individuals with lower $E(i)$. In all our simulations the mean $E(i)$ of individuals infected in a second wave was always significantly smaller than the mean of individuals infected in the first wave. Although we did not find systematic analysis examining this in the Covid-19 data, some anecdotal accounts suggest that the second wave affected those sectors of the society that remained protected in the 
first wave (Mallapaty 2021a, 2021b). Evidence for the acquired immunity being ineffective against the new variants is contradictory and therefore unreliable (Planas et al 2021, Powels et al 2021, Gazit et al 2021). In any case it is certainly not completely ineffective.

In case the new variant is only partially susceptible to prior acquired immunity, the binary immunity model is inappropriate to accommodate this possibility and our model needs to be brought in. The moment the continuous immunity model is invoked, it brings in its own intrinsic pattern of repeated waves, which should form the null model against which the new variant hypothesis should be tested. Since new variants keep on arising and their relative frequencies may drift or get selected by any mechanism of competition between viruses, the association of a variant with a new wave could only reflect a coincident hitch hiking on a wave. This null hypothesis can be rejected if a consistent correlation is shown between the increasing frequency of a variant and increasing $\mathrm{R}$ of the incidence curve across different countries or populations. Awaiting such a critical testing of the new variant hypothesis, at present both the alternative (but not mutually exclusive) hypotheses need to be kept open. It is also possible that a new variant gets selected by the altered immunity landscape of the host population. Since the immune response of a host is proportional to the intensity of invasion, it is likely that more invasive variants get selected when the host immunity is low (Shinde et al 2021). Therefore SIE is also likely to be causal to selection of more infectious variants. A critical question to ask is whether appearance of new variants is mutation limited or selection limited. It is possible to address this question with retrospective data. If it is mutation limited, we should see most new variants appearing near the peak of waves when viral populations are at their maxima. If selection limited, extended NPIs will be associated with more infective variants. Thus NPI, selection on new variants and small immunity effects are likely to be intertwined threads whose effects are difficult to segregate from each other.

Apart from the academic implications of our model in rethinking of the modelling approach, there are direct public health implications as well. The possible trade-off between short term and long term effects of PRs and the non-monotonic outcomes caution against blanket recommendation of lockdowns as well as personal protective measures over a long term. The measures that have a large social and economic cost should not be recommended since their outcomes are context dependent and at times turn counterproductive. Where and when it will 
turn counterproductive cannot be predicted very well at present. Therefore umbrella recommendations of such measures should not be done. In fact, this was the stand taken in a WHO report published a few months before the beginning of the pandemic (WHO 2019). But the cautionary note against implementation of the socially and economically costly NPIs appears to have been forgotten under the panic response to the pandemic. After vaccination, repeated exposure is most likely to boost and maintain long term immunity (Leino 2000). Therefore according to our model, other personal protection measures after vaccination should be contraindicated. At least substantial rethinking is required about public health policies to control infectious disease epidemics. One major hurdle in this has been lack of empirical studies on the small immunity effects, which is a hen and egg problem. Since the importance of SIEs is not appreciated, there is little motivation for empirical studies and since there is no data, modelling involving them fails to progress beyond a limit. At present we don't even have tools to monitor the small immunity changes at a population level. Antibody titres do not reflect all mechanisms of immunity. Population screening tools for other subtle mechanisms have not been developed. Therefore they are not incorporated in the current epidemiological thinking. We have tried to break this vicious cycle by indicating that at least theoretically the SIEs can alter the course of an epidemic substantially. The change is fundamental because what is currently assumed to limit the incidence may possibly be increasing it in the long run, as suggested by the model. At the minimum, the message of the gray immunity modelling exercise is that the factors assumed to be small and unimportant need more attention since they can potentially change public health policies fundamentally.

Acknowledgement: We thank Prof. Niranjan Joshi for critical comments on an earlier draft of the manuscript, Sonali Shinde for help in preparation of the manuscript.

\section{References:}

1. Abaluck J, Kwong L H, Styczynski Ashley, Haque A. et al. 2021. The Impact of Community Masking on COVID-19: A Cluster-Randomized Trial in Bangladesh. Clinical Trials. gov Identifier: NCT04630054

2. Agrawal M, Kanitkar M, Vidyasagar M. 2021. Modelling the spread of SARS-CoV-2 pandemic - Impact of lockdowns \& interventions. Indian J Med Res. 153(1 \& 2):175-181. doi: 10.4103/ijmr.IJMR_4051_20. PMID: 33146155; PMCID: PMC8184064. 
3. Alfano V., Ercolano S. 2020. The efficacy of lockdown against COVID-19: A cross country panel analysis Appl Health Econom Health Policy. 18(4):509-517.

4. Anttila J, Ruokolainen L, Kaitala V, Laakso J. 2013. Loss of Competition in the Outside Host Environment Generates Outbreaks of Environmental Opportunist Pathogens. PLoS ONE 8(8): e71621. doi:10.1371/journal.pone.0071621

5. Atalan A. 2020. Is the lockdown important to prevent the COVID-19 pandemic? Effects onpsychology, environment and economy-perspective Ann Med Surg, 56, 38-42.

6. Brauner JM. 2020. Inferring the effectiveness of government interventions against COVID-19Science; 371: 6531.

7. Carcione JM, Santos JE, Bagaini C, Ba J. 28May 2020. A Simulation of a COVID-19 Epidemic Based on a Deterministic SEIR Model. Front Public Health.;8:230. doi: 10.3389/fpubh.2020.00230. PMID: 32574303; PMCID: PMC7270399.

8. Cohen R, Ashman M, Taha MK, Varon E, Angoulvant F, Levy C, Rybak A, Ouldali N, Guiso N, Grimprel E. 2021. Pediatric Infectious Disease Group (GPIP) position paper on the immune debt of the COVID-19 pandemic in childhood, how can we fill the immunity gap? Infect Dis Now.51(5):418-423. doi: 10.1016/j.idnow.2021.05.004. Epub 2021, 12. PMID: 33991720; PMCID: PMC8114587.

9. Cooper I, Mondal A, 2020. Antonopoulos CG. A SIR model assumption for the spread of COVID-19 in different communities. Chaos Solitons Fractals.;139:110057. doi: 10.1016/j.chaos.2020.110057. Epub 2020 Jun 28. PMID: 32834610; PMCID: PMC7321055.

10. Doshi P. 2020. Covid-19: Do many people have pre-existing immunity? BMJ.;370:m3563. doi: 10.1136/bmj.m3563. PMID: 32943427.

11. Epstein JM, Hatna E, Crodelle J. Aug 4, 2021. Triple contagion: a two-fears epidemic model. J R Soc Interface. 2021 Aug;18(181):20210186. doi: 10.1098/rsif.2021.0186. Epub. PMID: 34343457; PMCID: PMC8331242.

12. Fudolig M, Howard R. 2020. The local stability of a modified multi-strain SIR model for emerging viral strains. PLoS ONE 15(12): e0243408. https://doi.org/10.1371/journal.pone.0243408 
13. Gadagkar SR. Jan-Feb 2015. Call GB. Computational tools for fitting the Hill equation to dose-response curves. J PharmacolToxicol Methods.;71:68-76. doi: 10.1016/j.vascn.2014.08.006. Epub 2014 Aug 23. PMID: 25157754.

14. GazitSivan, Roei Shlezinger, Galit Perez, Roni Lotan, Asaf Peretz, Amir BenTov, Dani Cohen, Khitam Muhsen, Gabriel Chodick, Tal Patalon. 2021. Comparing SARS-CoV-2 natural immunity to vaccine-induced immunity: reinfections versus breakthrough infections. medRxiv 2021.08.24.21262415; doi: https://doi.org/10.1101/2021.08.24.21262415

15. Giordano G, Blanchini F, Bruno R, Colaneri P, Di Filippo A, Di Matteo A, Colaneri M. 2020. Modelling the COVID-19 epidemic and implementation of population-wide interventions in Italy. Nat Med. 26(6):855-860. doi: 10.1038/s41591-020-0883-7. Epub 2020 Apr 22. PMID: 32322102; PMCID: PMC7175834.

16. Gold S, Donnelly CA, Woodroffe R, Nouvellet P (2021) Modelling the influence of naturally acquired immunity from subclinical infection on outbreak dynamics and persistence of rabies in domestic dogs. PLoS Negl Trop Dis 15(7): e0009581. https://doi.org/10.1371/journal.pntd.0009581

17. Haridas A. and Prathap G. 2020 The H1N1 factor and inverse correlation between death rates due to COVID-19 and influenza-pneumonia. Curr Sci 119, 535-539.

18. Humphreys D, ElGhazaly M, Frisan T. Senescence and Host-Pathogen Interactions. Cells. 2020 Jul 21;9(7):1747. doi: 10.3390/cells9071747. PMID: 32708331; PMCID: PMC7409240.

19. Hollingsworth B, Okamoto KW, Lloyd AL. 2020. After the honeymoon, the divorce: Unexpected outcomes of disease control measures against endemic infections. PLoSComput Biol 16(10): e1008292. https://doi.org/10.1371/journal.pcbi.1008292

20. Huang QS, Wood T, Jelley L, Jennings T, Jefferies S, Daniells K, Nesdale A, Dowell T, Turner N, Campbell-Stokes P, Balm M, Dobinson HC, Grant CC, James S, Aminisani N, Ralston J, Gunn W, Bocacao J, Danielewicz J, Moncrieff T, McNeill A, Lopez L, Waite B, Kiedrzynski T, Schrader H, Gray R, Cook K, Currin D, Engelbrecht C, Tapurau W, Emmerton L, Martin M, Baker MG, Taylor S, Trenholme A, Wong C, Lawrence S, McArthur C, Stanley A, Roberts S, Rahnama F, Bennett J, Mansell C, Dilcher M, Werno A, Grant J, van der Linden A, Youngblood B, Thomas PG. 2021. Impact of the COVID- 
19 nonpharmaceutical interventions on influenza and other respiratory viral infections in New Zealand. Nat Commun.;12(1):1001. doi: 10.1038/s41467-021-21157-9. PMID: 33579926 ; PMCID: PMC7881137.

21. Kharate, R.; Watve, M. 2021. Covid 19: Did Preventive Restrictions Work?. Preprints, 2021040286 (doi: 10.20944/preprints202104.0286.v1).

22. Kharroubi S and Saleh F. 2020. Are lockdown measures effective against COVID-19? Front. Pub Health; 8:549692.

23. Kieran Dee, Daniel M Goldfarb, Joanne Haney, Julien A R Amat, Vanessa Herder, Meredith Stewart, Agnieszka M Szemiel, Marc Baguelin, Pablo R Murcia. 2021. Human Rhinovirus Infection Blocks Severe Acute Respiratory Syndrome Coronavirus 2 Replication Within the Respiratory Epithelium: Implications for COVID-19 Epidemiology, The Journal of Infectious Diseases, Volume 224, Issue 1, Pages 3138, https://doi.org/10.1093/infdis/jiab147

24. Krammer F. 2021. Correlates of protection from SARS-CoV-2 infection. Lancet.397(10283):1421-1423. doi: 10.1016/S0140-6736(21)00782-0. Epub 2021 Apr 9. PMID: 33844964; PMCID: PMC8040540.

25. Leino T, Auranen K, Mäkelä PH, Käyhty H, Takala AK. 2000. Dynamics of natural immunity caused by subclinical infections, case study on Haemophilus influenzae type $\mathrm{b}$ (Hib). Epidemiol Infect.;125(3):583-91. doi: 10.1017/s0950268800004799. PMID: 11218209; PMCID: PMC2869642.

26. Leung T, Campbell PT, Hughes BD, Frascoli F, McCaw JM. 2018. Infection-acquired versus vaccine-acquired immunity in an SIRWS model. Infect Dis Model. 3:118-135. doi: 10.1016/j.idm.2018.06.002. PMID: 30839933; PMCID: PMC6326260.

27. Long QX, Tang XJ, Shi QL, Li Q, Deng HJ, Yuan J, Hu JL, Xu W, Zhang Y, Lv FJ, Su K, Zhang F, Gong J, Wu B, Liu XM, Li JJ, Qiu JF, Chen J, Huang AL. 2020. Clinical and immunological assessment of asymptomatic SARS-CoV-2 infections. Nat Med.26(8):1200-1204. doi: 10.1038/s41591-020-0965-6. Epub 2020 Jun 18. PMID: 32555424.

28. Mallajosyula V, Ganjavi C, Chakraborty S, McSween AM, Pavlovitch-Bedzyk AJ, Wilhelmy J, Nau A, Manohar M, Nadeau KC, Davis MM. 2021. CD8 ${ }^{+}$T cells specific 
for conserved coronavirus epitopes correlate with milder disease in COVID-19 patients. Sci Immunol.6(61):eabg5669. doi: 10.1126/sciimmunol.abg5669. PMID: 34210785.

29. Mallapaty S. 2021. India's massive COVID surge puzzles scientists. Nature. .592(7856):667-668. doi: 10.1038/d41586-021-01059-y. PMID: 33883710.

30. Mallapaty S. 2021. Delta threatens rural regions that dodged earlier COVID waves. Nature. b Aug;596(7872):325-326. doi: 10.1038/d41586-021-02146-w. PMID: 34354278.

31. Marriott D, Beresford R, Mirdad F, Stark D, Glanville A, Chapman S, Harkness J, Dore GJ, Andresen D, Matthews GV. 2021. Concomitant Marked Decline in Prevalence of Severe Acute Respiratory Syndrome Coronavirus 2 (SARS-CoV-2) and Other Respiratory Viruses Among Symptomatic Patients Following Public Health Interventions in Australia: Data from St Vincent's Hospital and Associated Screening Clinics, Sydney, NSW. Clin Infect Dis.72(10):e649-e651. doi: 10.1093/cid/ciaa1256. PMID: 32841316; PMCID: PMC7499558.

32. McLean AR, Bostock CJ. 2000. Scrapie infections initiated at varying doses: an analysis of 117 titration experiments. Philos Trans R Soc Lond B Biol Sci. 355(1400):1043-50. doi: 10.1098/rstb.2000.0641. PMID: 11186305; PMCID: PMC1692811.

33. Moein, S., Nickaeen, N., Roointan, A. et al. 2021. Inefficiency of SIR models in forecasting COVID-19 epidemic: a case study of Isfahan. Sci Rep 11, 4725. https://doi.org/10.1038/s41598-021-84055-6

34. Ng KW, Faulkner N, Cornish GH, Rosa A, Harvey R, Hussain S, Ulferts R, Earl C, Wrobel AG, Benton DJ, Roustan C, Bolland W, Thompson R, Agua-Doce A, Hobson P, Heaney J, Rickman H, Paraskevopoulou S, Houlihan CF, Thomson K, Sanchez E, Shin GY, Spyer MJ, Joshi D, O'Reilly N, Walker PA, Kjaer S, Riddell A, Moore C, Jebson BR, Wilkinson M, Marshall LR, Rosser EC, Radziszewska A, Peckham H, Ciurtin C, Wedderburn LR, Beale R, Swanton C, Gandhi S, Stockinger B, McCauley J, Gamblin SJ, McCoy LE, Cherepanov P, Nastouli E, Kassiotis G. 2020. Preexisting and de novo humoral immunity to SARS-CoV-2 in humans. Science. 370(6522):1339-1343. doi: 10.1126/science.abe1107. Epub 2020 Nov 6. PMID: 33159009; PMCID: PMC7857411. 
35. Planas, D., Veyer, D., Baidaliuk, A. et al. 2021. Reduced sensitivity of SARS-CoV-2 variant Delta to antibody neutralization. Nature 596, 276-280. https://doi.org/10.1038/s41586-021-03777-9

36. Pouwels, K. B. et al.2021. Preprint at Univ. Oxford https://www.ndm.ox.ac.uk/files/coronavirus/covid-19-infectionsurvey/finalfinalcombinedve20210816.pdf

37. Read, A. F., 1994. The evolution of virulence. Trends Microbiol., 2,73-76

38. Regoes RR Ebert D and Bonhoffer S. 2002.Dose-dependent infection rates of parasites produce an Allee effect in Epidemiology Proc. R. Soc. Lond. B. 269, 271-279

39. Sanderson K. 2021. COVID vaccines protect against Delta, but their effectiveness wanes. Nature news. https://www.nature.com/articles/d41586-021-02261-8

40. Santos Rocha C, Hirao LA, Weber MG, Méndez-Lagares G, Chang WLW, Jiang G, Deere JD, Sparger EE, Roberts J, Barry PA, Hartigan-O'Connor DJ, Dandekar S. 2018. Subclinical Cytomegalovirus Infection Is Associated with Altered Host Immunity, Gut Microbiota, and Vaccine Responses. J Virol.92(13):e00167-18. doi: 10.1128/JVI.0016718. PMID: 29669841; PMCID: PMC6002712.

41. Sette A, Crotty S. 2020. Pre-existing immunity to SARS-CoV-2: the knowns and unknowns. Nat Rev Immunol. 20(8):457-458. doi: 10.1038/s41577-020-0389-z. Erratum in: Nat Rev Immunol., 20(10):644. PMID: 32636479; PMCID: PMC7339790.

42. Shah, K., D Saxena, D Mavalankar, 2020. Secondary attack rate of COVID-19 in household contacts: a systematic review, QJM: An International Journal of Medicine, 113(12), 841-850, https://doi.org/10.1093/qjmed/hcaa232

43. Shinde S, Ranade P, Watve M. 2021. Evaluating alternative hypotheses to explain the downward trend in the indices of the COVID-19 pandemic death rate. PeerJ9:e11150 DOI 10.7717/peerj.11150.

44. Tang L, Zhou Y, Wang L, et al. 2020. A Review of multi-compartment infectious disease models. Int Stat Rev.;88(2):462-513. doi:10.1111/insr.12402

45. Tolles J, Luong T. 2020. Modeling epidemics with compartmental models. JAMA. 323(24):2515-2516. doi:10.1001/jama.2020.8420

46. Turner JS, Kim W, Kalaidina E, Goss CW, Rauseo AM, Schmitz AJ, Hansen L, Haile A, Klebert MK, Pusic I, O'Halloran JA, Presti RM, Ellebedy AH. 2021. SARS-CoV-2 
infection induces long-lived bone marrow plasma cells in humans. Nature. 595(7867):421-425. doi: 10.1038/s41586-021-03647-4. Epub 2021 May 24. PMID: 34030176.

47. Whittle HC, Aaby P, Samb B, Jensen H, Bennett J, Simondon F. 1999. Effect of subclinical infection on maintaining immunity against measles in vaccinated children in West Africa. Lancet. 9;353(9147):98-102. doi: 10.1016/S0140-6736(98)02364-2. PMID: 10023894.

48. World Health Organization. Non-pharmaceutical public health measures for mitigating the risk and impact of epidemic and pandemic influenza; 2019. Licence: CC BY-NC-SA 3.0 IGO. https://apps.who.int/iris/handle/10665/329439 